\title{
EWSR1/DDIT3 Fusion Gene
}

National Cancer Institute

\section{Source}

National Cancer Institute. EWSR1/DDIT3 Fusion Gene. NCI Thesaurus. Code C99200.

A fusion gene that results from a chromosomal translocation $t(12 ; 22)(q 13 ; p 11)$ which

fuses either the first 7 or the first 10 exons of the EWSR1 gene with exon 2 of the DDIT 3 gene. This rearrangement is associated with myxoid liposarcoma. 